\title{
Correspondence
}

\section{Prelaparotomy diagnosis of extrahepatic biliary atresia}

Sir,

We were interested in the conclusion of Manolaki and his colleagues $^{1}$ on the diagnostic value of gammaglutamyl transpeptidase (GGT) which was in contrast to other reports on this subject. ${ }^{2-4}$ We suggest that the analysis of the data has both methodological and statistical flaws.

In the report of Manolaki et al, the GGT concentrations in both groups were obviously not normally distributed as there was a positive skewing (mean (SD), extrahepatic biliary atresia (EHBA), 975 (518.1) IU/1; intrahepatic disease (IHD), 454 (458) IU/l) and hence the application of Student's $t$ test was inappropriate. Either the data should have been transformed or a non-parametric statistical method should have been used; otherwise the 'normal' range of GGT concentrations for either group could not have been defined confidently.

From a statistical point of view it is more reasonable to take the mean of two medians as a cut off value for the differentiation of EHBA from IHD. Experience from our study $^{5}$ of 21 cases of EHBA (median, $1140 \mathrm{IU} / 1$; mean (SD), 1112 (607) IU/l) and 20 cases of IHD (median, 239 IU/l; mean (SD), 275 (195) IU/l) showed that by using the mean (690 IU/l) of the medians of these two groups, the sensitivity and specificity of GGT were $78.9 \%$ and $95.2 \%$ respectively. This compares favourably with the accuracy of the more invasive procedure of liver biopsy. ${ }^{1}$

We found that the enzyme concentrations at presentation were appreciably lower than the subsequent readings in the EHBA group, and therefore the highest reading available should be taken for statistical analysis. There was a correlation between the enzyme value and the duration of disease in the EHBA group of patients $(P=0.05$, rank correlation test) and this feature may be utilised for differentiation. Manolaki et al analysed the fractional change in enzyme activity. When there is a low initial and a small subsequent change in GGT activity, its fractional change may be grossly exaggerated and misleading. In their report the time period within which the change in GGT activity was measured was not clear. The absolute rate of rise of GGT should be analysed instead of the fractional change. In our series serial measurement in 9 patients with EHBA exhibited a rapid rise in GGT activity (median=17.1 IU/V/day, range 9.4-246 IU/V/day) but the rate of rise in 11 of 12 patients with IHD was significantly lower (median $=1.5 \mathrm{IU} / \mathrm{l} /$ day, range -7 to $15.9 \mathrm{IU} / \mathrm{l} /$ day, $\mathbf{P}=0.0021$ ). We therefore suggest that the rate of rise is more diagnostic than a single measurement of the GGT concentration. Repeated measurements during the first two weeks after presentation, which is within the normal duration of diagnostic 'workup', could serve as a prelaparotomy, non-invasive screening test.

We concur with the authors that the aspartate aminotransferase (AST) titres fluctuated widely and no consis- tent pattern was detected in either group. The GGT/AST ratio is detrimental therefore to the differential diagnosis unless the AST titres of the IHD group are significantly higher than those of the EHBA group

S P LAU AND K P Fung Paediatric Department, Queen Mary Hospital, University of Hong Kong, Hong Kong

\section{References}

${ }^{1}$ Manolaki AG, Larcher VF, Mowat AP, et al. The prelaparotomy diagnosis of extrahepatic biliary atresia. Arch Dis Child 1983;58:591-4.

2 Wright K, Christie DL. Use of gamma-glutamyl transpeptidase in the diagnosis of biliary atresia. Am J Dis Child 1981;135: 134-6.

3 Platt MS, Potter J, Bockman CR, Jaberg C. Elevated GGTP/ SGOT ratio. An early indicator of infantile obstructive cholangiopathy. Am J Dis Child 1981;135:834-6.

4 Vasquez C, Fidalgo I, Busturia I, Mondragon F. Gammaglutamyl transpeptidase and infantile biliary obstruction. (Letter). Am J Dis Child 1982;136:755.

5 Fung KP, Lau SP. Gamma-glutamyltranspeptidase activity and its serial measurement in differentiation between extrahepatic biliary atresia and neonatal hepatitis. Acta Paediatr Scand; In press.

Drs Mowat and Manolaki comment:

Since submitting our paper our experience with serum gammaglutamyl transpeptidase (GGT) values in extrahepatic biliary atresia (EHBA) and intrahepatic diseases causing conjugated hyperbilirubinaemia in infancy (IHD) has emphasised the total unreliability of single GGT value in distinguishing these conditions in the individual patient. Three patients aged less than 16 weeks with surgically and pathologically confirmed EHBA have been seen with GGT values lower than the 162 IU/l which was the lowest value found in the patients studied in paper. One had a GGT concentration in the normal range of $\mathbf{4 2}$ IU/l. There is no doubt that the mean concentration of GGT in EHBA is higher than in IHD. We have no wish to argue the relative merits of the statistical methods which may be used to show this. The scatter of GGT values in the patients in our study is very similar to that published for the GGT:aspartate aminotransferase ratio in the Figure in our paper. Irrespective of what cut off value of GGT we derive from statistical methods, we would be depriving some patients with EHBA from the possible benefits of surgery or doing an unacceptably high level of unnecessary laparotomies in patients with IHD.

With respect to serial studies, we apologise for not stating the time period during which preoperative GGT values were studied in patients with biliary atresia. This was determined by the time taken to reach a definitive diagnosis and to submit the patient to surgery. In the vast majority of patients it was less than two weeks, but 